\title{
СИТУАЦИОННО-СИНЕРГЕТИЧЕСКИЙ ПОДХОД К АККУМУЛЯЦИИ ЗНАНИЙ В СИСТЕМАХ УПРАВЛЕНИЯ ПРОМЫШЛЕННЫМ ПРЕДПРИЯТИЕМ
}

\author{
(c) 2019 Жук Марина Алексеевна \\ доктор экономических наук, доцент, \\ заведующий кафедрой прикладной информатики в экономике и управлении \\ Оренбургский государственный университет, Россия, Оренбург \\ E-mail: eng_m@inbox.ru
}

В статье представлены результаты исследования в области совершенствования механизмов управления предприятиями за счет внедрения систем информационной поддержки принятия решений, основанных на знаниях, аккумулируемых и генерируемых в автоматическом режиме. За основу взяты ситуационный и синергетический подходы к формированию информационной инфраструктуры управления.

Ключевые слова: промышленное предприятие; информационная инфраструктура; проблемная ситуация управления; база знаний; аккумуляция знаний.

В настоящее время повышение эффективности работы предприятий промышленного комплекса является актуальной проблемой, так как в условиях импортозамещения качество продукции подобных предприятий и степень удовлетворения потребительского спроса играют значительную роль в обеспечении качества жизни населения. В условиях цифровизации экономических процессов наиболее перспективным направлением в аспекте повышения эффективности регионального промышленного комплекса является интенсификация сетевых взаимодействий между предприятиями и, как следствие, образование кластеров, ориентированных на решение проблем экономического развития региона.

Поскольку взаимодействие хозяйствующих субъектов все больше переносится в виртуальную среду (глобальное информационное пространство) процессы интеграции предприятий в сетевые сообщества напрямую зависят от качества работы корпоративных информационных систем предприятия. В современных реалиях корпоративная информационная система должна оказывать всестороннюю информационную поддержку принятия управленческих решений, в связи с этим особую актуальность приобретают исследования, лежащие в плоскости разработки интеллектуальных информационных систем, позволяющих максимально исключить долю участия человека в процессе принятия решения, устранить субъективную составляющую, эффективно использовать накопленный корпо- ративный управленческий опыт.

Несмотря на широту и многообразие исследований в данной области, не теряет своей актуальности проблема создания методик автоматического накопления и актуализации знаний. Целью настоящего исследования стала разработка механизма генерации фрагментов знаний, аналогичных экспертным, на основе синергетической оценки информации, аккумулируемой в процессе выполнения управленческих функций.

Проблемы совершенствования механизмов управления промышленными предприятиями в настоящее время лежат в плоскости цифровизации региональных экономических процессов. В связи с этим, одним из путей повышения эффективности развития регионального промышленного комплекса является формирование интеграционных образований, по сути, являющихся кластерами. В источниках $[1,5]$ рассматриваются вопросы реализации механизмов сетевого взаимодействия предприятий и получения синергетических эффектов от их совместного взаимодействия, указанные источники были использованы в процессе исследования.

Сетевое взаимодействие предприятий невозможно без интенсификации процессов информатизации промышленных предприятий на всех уровнях управления. Авторами проанализирован ряд источников, содержащих опыт внедрения информационных систем в процесс управления предприятиями. В современных условиях акцент делается на изучение информационных потоков, приводится анализ влияния 
показателей продукта, информации и денежных потоков на управление цепочками поставок и т.д. Накопленный опыт показывает, что цифровизация промышленных предприятий позволяет обеспечить рост продуктивности, повышение конкурентоспособности, соответствие принципам рационального природопользования и охраны природы.

Современный уровень развития информационно-коммуникационных технологий предоставляет дополнительные возможности для развития цифровой экономики и цифрового общества. В таких условиях определяются тенденции к формированию экономики знаний («knowledge economy»), которая определяется как «концепция экономического развития, при которой инновации и доступ к информации стимулируют рост производительности труда» [5]. Согласно данным Европейского банка реконструкции и развития Россия занимает семнадцатую строчку рейтинга с общим счетом 4 ,93. Такое значение относит Россию к кластеру со средним уровнем развития экономики знаний. Улучшение значений показателей позволит России получать дополнительное финансирование проектов, направленных на экономическое развитие. Переход к экономике знаний в России связан с активным внедрением цифровых технологий, применение которых способствует повышению производительности, ускорению процессов взаимодействия участников экономических отношений, повышению конкурентоспособности на мировом рынке.

В цифровых технологиях особое место занимают технологии аккумуляции знаний или управления знаниями. Под аккумуляцией знаний понимаются процессы извлечения, структуризации, хранения и использовании знаний в процессе принятия управленческих решений. Совершенствование информационных систем управления за счет внедрения интеллектуальных подсистем поддержки принятия решений, основанных на обработке знаний, инициирует проблему дополнения и актуализации баз знаний в автоматическом режиме. В этом аспекте особый интерес представляют работы [2] и [7], раскрывающие соответственно объектно-ориентированный и процессноориентированный подходы к проблемам аккумуляции знаний.

Исследования проводились на основе анализа предприятий Оренбургской области. Соглас- но намеченным направлениям регионального развития, предприятия области с каждым годом наращивают инфо-коммуникационные мощности, только за последние три года уровень информатизации областных предприятий вырос на 58\%, в процессе управления предприятиями широко используются учетно-аналитические системы управления, разработанные на платформе 1С. Взаимодействие предприятий как хозяйствующих субъектов (экономических агентов) все в большей степени переносится в виртуальную среду посредством реализации бизнес-процессов в он-лайн режиме, что значительно ускоряет процессы взаимодействия и способствует интеграции промышленных предприятий в сетевые структуры для совместной реализации программ регионального развития.

Очевидно, что необходимо наличие устойчивой информационной инфраструктуры, обеспечивающей процессы взаимодействия региональных предприятий в глобальном информационном пространстве, при этом благодаря использованию в процессе управления предприятием информационной системы на локальном уровне такие инфраструктура существуют для каждого предприятия.

В исследовании для разработки концептуальной модели информационной инфраструктуры взаимодействия региональных предприятий использовался ситуационный подход [3], согласно которому в качестве базовой единицы моделирования рассматривается проблемная ситуация управления (ПСУ). Для формирования первичных структур аккумуляции знаний использован мультиагентный подход [6], позволяющий рассматривать региональные предприятия как агентов, автономной системой управления каждого из которых является информационная система предприятия, а информационная инфраструктура их взаимодействия как мультиагентная среда.

В качестве базовой выбрана методология мультиагентной архитектуры среды взаимодействия региональных предприятий, представленная в [4], основанная на использовании фреймово-продукционной модели представления знаний.

Структуры аккумуляции знаний ориентированы на накопление фактографической и экспертной информации, характеризующей отработанные управленческие решения. Согласно специфике фреймово-продукционной модели 
аккумулируемая часть знаний - это продукции, построенные по классической причинноследственной схеме «антецедент $\rightarrow$ консеквент». Для генерации продукций, как элементов базы знаний, используется синергетический подход, согласно которому накапливаемая в вышеуказанных структурах информация оценивается на предмет выявления «устойчивых управленческих стратегий» и трансформируется в готовую продукцию, как полноценный элемент базы знаний

В результате последовательной декомпозиции по уровням детализации построена структурная схема базовых ПСУ промышленных предприятий. Полученная в результате декомпозиции схема ПСУ представляет собой граф, терминальными вершинами которого являются ПСУ, проиндексированные по уровням иерархии, то есть имеющими составной индекс, для которого введем обозначение - $\mathrm{p}_{\mathrm{k} 1 \mathrm{~m}}$, все множество терминальных вершин обозначим $\mathrm{p}^{\text {term }}=\left\{\mathrm{p}_{\mathrm{k} 1 \mathrm{~m}}\right\}$.Так как далее речь будет идти только о терминальных вершинах для упрощения формализмов составной индекс можно свести к одноместному обозначению $\mathrm{p}_{\mathrm{z}}, \mathrm{z}=\overline{1, Z}$, где $\mathrm{Z}$ - количество элементов множества $\mathrm{p}^{\text {term }}$

Согласно методологии [4] мультиагентная модель взаимодействия промышленных предприятий в глобальном информационном пространстве представлена набором полюсов акку-

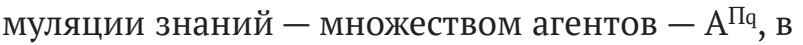
предлагаемой модели каждый агент интерпретируется как региональное предприятие, а точнее его корпоративная информационная система, встроенная в инфраструктуру виртуального взаимодействия предприятий, $\mathrm{q}=\overline{1, Q}$, где $\mathrm{Q}$ - общее количество предприятий.

Так как в модели принимается допущение о том, что все агенты $\mathrm{A}^{П q}$ имеют одинаковую базовую структуру, целесообразно обозначение $\mathrm{A}^{\Pi}$. Базовая структура $\mathrm{A}^{\Pi}$ состоит из набора функциональных объектов (информационных подсистем) $\mathrm{A}^{\Pi} \rightarrow \mathrm{O}\left\{\mathrm{O}_{\mathrm{i}}\right\}, \mathrm{O}_{\mathrm{i}}-\mathrm{i}$-тый объект, $\mathrm{i}=\overline{1, \mathrm{~N}}, \mathrm{~N}$ - количество объектов, поведение агента может быть описано посредством набора бизнес-процессов: $B_{i}=\left\{F_{i j}\right\}, F_{i j}-j$-тая функция i-того бизнес-процесса, $\mathrm{i}=\overline{1, \mathrm{~K}}, \mathrm{j}=\overline{1, \mathrm{~L}_{\mathrm{i}}}$ $\mathrm{K}$ - процессов, $\mathrm{L}_{\mathrm{i}}$ - количество функций в $\mathrm{i}-\mathrm{Toм}$ процессе. Объект $\mathrm{O}_{\mathrm{i}}$ описывается атрибутами $\mathrm{O}_{\mathrm{i}}=<\mathrm{NamO}_{\mathrm{i}}, \mathrm{AtrO}_{\mathrm{i}}, \mathrm{ParO}_{\mathrm{i}}>, \mathrm{NamO}_{\mathrm{i}}$ - заголовок $\mathrm{O}_{\mathrm{i}}$; $\mathrm{AtrO}_{\mathrm{i}}$ - множество атрибутов $\mathrm{O}_{\mathrm{i}}$, описываемое как: $\operatorname{AtrO}_{\mathrm{i}}=\left\{\mathrm{AtrO}_{\mathrm{ij}}, \mathrm{Zn}_{\mathrm{ij}}\right\}, \mathrm{AtrO}_{\mathrm{ij}}$ - имя j-го атри- бута $\mathrm{O}_{\mathrm{i}}, \mathrm{j}={\overline{1, \mathrm{~N}_{\mathrm{i}}}}_{\mathrm{i}}, \mathrm{N}_{\mathrm{i}}-$ количество атрибутов; $\mathrm{Zn}_{\mathrm{ij}}-$ значение j-го атрибута $\mathrm{O}_{\mathrm{i}}$. Функция $\mathrm{F}_{\mathrm{ij}}$ - описывается кортежем $\mathrm{F}_{\mathrm{ij}}=<\mathrm{NameF}_{\mathrm{ij}}$, $\operatorname{Pred}_{\mathrm{ij}}$, Ind $_{\mathrm{ijr}}>$ $\mathrm{NameF}_{\mathrm{ij}}$ - заголовок функции; $\operatorname{Pred}_{\mathrm{ij}}$ - функция, предшествующая $\mathrm{F}_{\mathrm{ij}}$, Ind $\mathrm{Inj}_{\mathrm{r}}$ - r-тый показатель результативности.

С целью описания взаимодействия предприятий вводится понятие цепочки бизнес-процессов, связь между процессами описывается по типу «источник-приемник»- FIs $\mathrm{s}_{\mathrm{i}}$ - процесс-источник, $\mathrm{FPr}_{\mathrm{i}}$ - процесс-приемник. Согласно выбранной методологии существует объект-коннектор ObjConnect $_{\mathrm{i}}=<\mathrm{NameO}_{\mathrm{i}}, \mathrm{FIs}_{\mathrm{i}}, \mathrm{FPr}_{\mathrm{i}}, \mathrm{RoleO}_{\mathrm{i}}>$ NameO $_{\mathrm{i}}-$ заголовок объекта-коннектора, $\mathrm{RoleO}_{\mathrm{i}}$ - роль i-той связи в процессе $\mathrm{FPr}_{\mathrm{i}}$. Предлагаемая мультиагентная модель позволяет создавать в инфраструктуре виртуального взаимодействия агентов структуры, аккумулирующие информацию об отработанных в результате взаимодействия бизнес-процессах.

Для реализации синергетического подхода аккумулируемая информация должна содержать оценку результативности бизнес-процесса, реализуемого в ходе виртуального взаимодействия агентов, значения оценки могут быть качественными, но для реализации алгоритмов обработки они должны быть переведены в количественные. С этой целью в модель вводятся два индикаторных элемента, выполняющих функцию аккумуляции - накопитель результатов бизнеспроцессов и накопитель актов взаимодействия агентов, модель представлена на рисунке 1 .

На рисунке изображено взаимодействие двух предприятий, агент $\mathrm{A}_{\text {Ист }}^{П}$ инициирует акт взаимодействия и вся информация, аккумулируемая для генерации знаний, накапливается в информационной системе этого

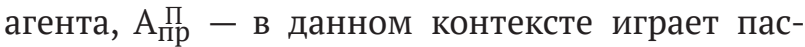
сивную роль. Компонента $\mathrm{k}_{\mathrm{B}_{\mathrm{ij}}}$ - это база продукций соответствующего бизнес-процесса, продукция рассматривается как импликация $\gamma_{m}\left(F_{1}\right) \wedge \ldots \wedge \gamma_{m}\left(F_{i}\right) \wedge \ldots \wedge \gamma_{m}\left(F_{l_{B_{m}}}\right) \rightarrow \phi_{m}\left(\rho_{j}\right)$, где $\mathrm{I}_{\mathrm{B}_{\mathrm{m}}}-$ число функций, входящих в состав $\mathrm{m}$-того бизнес-процесса. Индикатором завершения реализации бизнес-процесса выступает величина $\gamma_{m}\left(F_{i}\right) \in\{0,1\}$, соответственно - $0-$ процесс прерван, 1 - процесс успешно завершен. Величина $\phi_{m}\left(\rho_{j}\right)$ интерпретируется как оценка степени

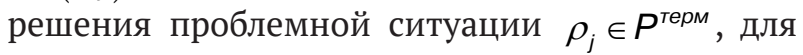
получения количественного значения оценка выставляется экспертно, шкала оценок может настраиваться. 


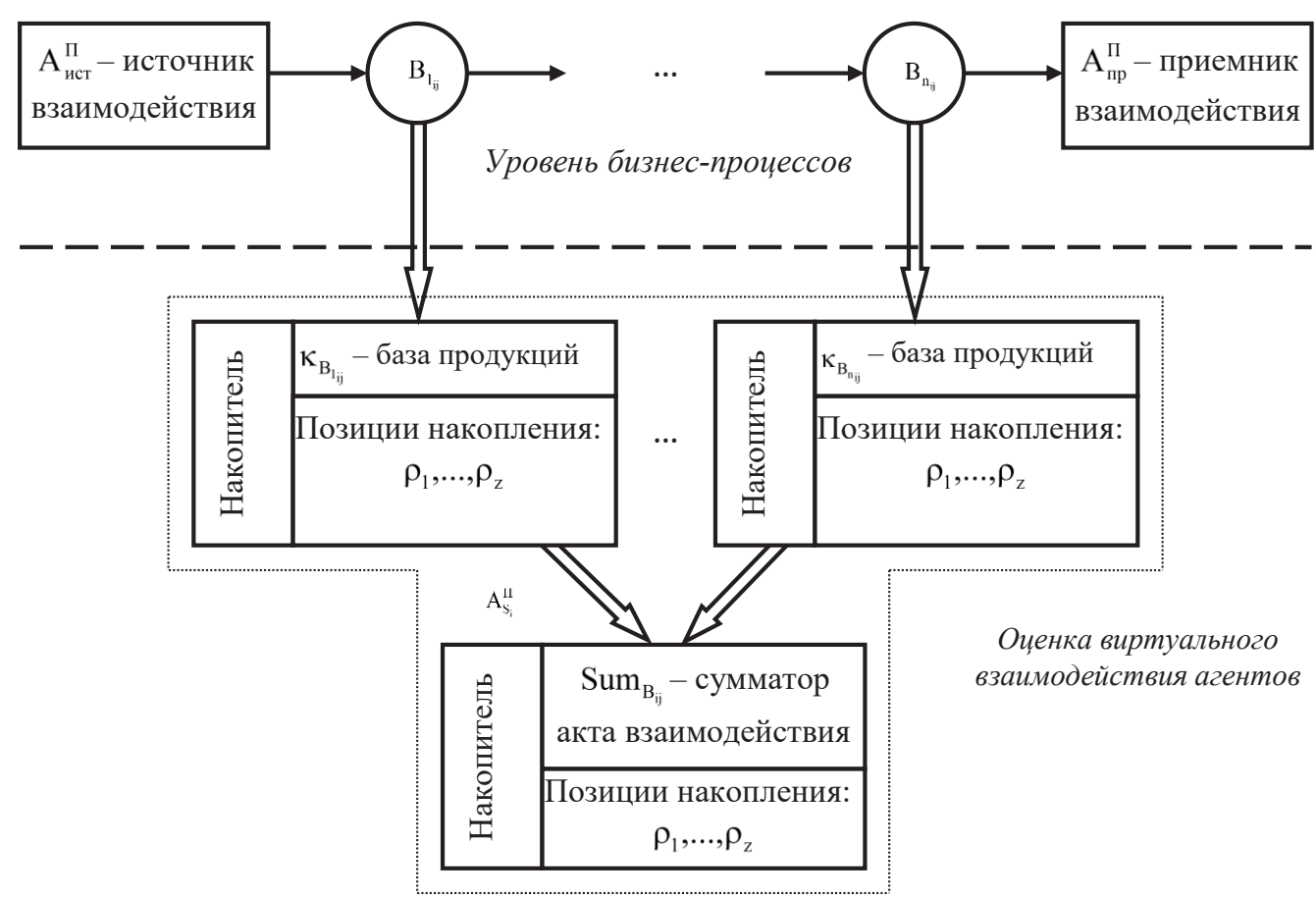

Puc. 1. Модель аккумуляции информации в процессе взаимодействия агентов

Возможный вариант:

$$
\varphi_{\mathrm{m}}\left(\rho_{\mathrm{j}}\right)=\left\{\begin{array}{c}
-1 \text { - однозначно отрицательно; } \\
-0,5 \text { - возможно отрицательно; } \\
0 \text { - не имеет отношение к данной ПСУ; } \\
0,5 \text { - возможно положительно; } \\
1 \text { - однозначно положительно. }
\end{array}\right.
$$

Таким образом, каждому бизнес-процессу, инициируемому в результате взаимодействия i-того и ј-того агентов, может быть поставлено в соответствие упорядоченное множество $B_{m_{i j}}=\left(\phi_{m}\left(\rho_{1}\right), \phi_{m}\left(\rho_{2}\right), \ldots, \phi_{m}\left(\rho_{z}\right)\right)$, значения поступают в соответствующий накопитель - сумматор $\operatorname{Sum}_{\mathrm{B}_{\mathrm{ij}}}$ механизм работы которого может быть реализован посредством аддитивной свертки по видам ПСУ:

$$
\begin{aligned}
& \operatorname{Sum}_{1_{\mathrm{B}_{\mathrm{ij}}}}=\sum_{\mathrm{m}=1}^{\mathrm{n}} \psi_{1_{\mathrm{m}}} \varphi_{\mathrm{m}}\left(\rho_{1}\right) ; \\
& \operatorname{Sum}_{\mathrm{DB}_{\mathrm{ij}}}=\sum_{\mathrm{m}=1}^{\mathrm{n}} \psi_{1_{\mathrm{m}}} \varphi_{\mathrm{m}}\left(\rho_{1}\right)
\end{aligned}
$$

$\mathrm{D}$ - количество видов ПСУ, величины $\psi_{1 \mathrm{~m}}$, $\mathrm{m}=\overline{1, \mathrm{~N}} ; 1=\overline{1, \mathrm{Z}}$ вводятся в модель как весовые коэффициенты по каждому бизнес-процессу по отношению к той или иной ПСУ. Для иден- тификации факта взаимодействия i-того и j-того агентов на временном интервале от $\mathrm{T}_{0}$ до Т вводится величина $A c t_{A^{n} A^{n}}^{T}$, эффект виртуального взаимодействия агентов описыва-

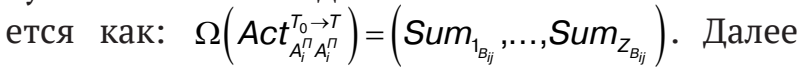
вводится пороговое значение частоты однотипных взаимодействий, накапливающих положительные оценки эффекта, тогда, согласно синергетическому подходу, в момент перехода порогового значения импликация $\gamma_{m}\left(F_{1}\right) \wedge \ldots \wedge \gamma_{m}\left(F_{i}\right) \wedge \ldots \wedge \gamma_{m}\left(F_{l_{B_{m}}}\right) \rightarrow \phi_{m}\left(\rho_{j}\right) \quad$ интерпретируется как полноценная продукция, которая может быть добавлена в базу знаний агента $\mathrm{A}_{\text {ист }}^{\text {П }}$

Предлагаемая модель аккумуляции знаний достаточно просто интегрируется в корпоративную информационную систему предприятия, фактически она представляет собой некоторую подсистему поддержки принятия решений, которая является частью корпоративной информационной системы. Одним из видов систем поддержки при принятии решений являются интеллектуальные информационные системы, основанные на обработке знаний экспертов. В предлагаемой модели знания экспертов заменяются автоматически аккумулируемыми продукционными правилами, что позволяет значительно ускорить процессы актуализации и 
пополнения баз знаний, осуществлять их фактически без участия персонала.

Однако представленный механизм содержит ряд допущений, предполагающих участие экспертов в работе системы. Так одним из них является процедура оценки степени разрешения проблемной ситуации посредством реализации бизнес-процессов. При проектировании интеллектуальной информационной системы представленная модель предполагает возможность настройки шкалы экспертных оценок для получения наиболее точных и адекватных результатов. В качестве перспективы развития предлагаемого механизма можно наметить исследования по разработке процедур автоматической оценки степени разрешения проблемных ситуаций, на основе формализации оценки наборов косвенных факторов, что является перспективным для дальнейших исследований в данном направлении.

В результате исследования получен механизм совершенствования процессов управления предприятиями регионального промышленного комплекса за счет информационной поддержки принятия решений, реализуемой интеллектуальными подсистемами, основанными на обработке знаний. При этом решается проблема получения знаний от экспертов, что является достаточно ресурсозатратным мероприятием, как по стоимости получения знаний, так и по времени, необходимому для их структуризации, обработке на непротиворечивость и переноса в базу. Решение проблемы осуществляется за счет предлагаемого двухступенчатого механизма автоматической генерации продукционных правил, состоящего из этапа аккумуляции информации о результативности бизнес-процессов, осуществляемых предприятиями в процессе их функционирования и формирования готового продукционного правила на основе синергетического подхода к оценке накапливаемой информации. Полученные результаты позволяют расширять и актуализовать базы знаний корпоративных информационных систем предприятий в автоматическом режиме.

\section{Библиографический список}

1. Ансофф, И. Стратегический менеджмент. Классическое издание.- С. Пб: Питер. 2009. - 344 с.1

2. Брусакова И.А., Сербин А.А. Объектно-ориентированный подход в моделях аккумуляции знаний о бизнеспроцессах // Сб. научных трудов научно-практической конференции по современным проблемам прикладной информатики, СПб: Изд-во Политехн. ун-та, 2008. С. 11-29.

3. Демидкина, А.М. Ситуационный подход в управлении предприятием // Проблемы экономики, 2007, № 4, С. 55-56.

4. ук М.А. Мультиагентное моделирование среды виртуального взаимодействия региональных субъектов // Управление экономическими системами: электронный научный журнал, 2011, № 32., С. 30.

5. Клейнер, Г.Б. Развитие теории экономических систем и ее применение в корпоративном стратегическом управлении // Центральный экономико-математический институт, Российская академия наук / Препринт, \#WP/99/2010. Москва.

6. Рассел С., Норвиг П. Искусственный интеллект: современный подход, М.: Издательский дом «Вильямс», 2006. - 1408 c.

7. Тельнов Ю. Ф. Реинжиниринг бизнес-процессов, М.: Финансы и статистика, 2004. - 314 с. 